\title{
Role of the posterior capsule in the prevention of postoperative bacterial endophthalmitis: experimental primate studies and clinical implications*
}

\author{
TODD L BEYER, FRANK E O'DONNELL, VALENTIM GONCALVES, \\ AND RAJKUMAR SINGH
}

From the Bethesda Eye Institute, Department of Ophthalmology, St Louis University School of Medicine, St Louis, Missouri, USA

SUMMARY The posterior capsule has an important effect on the risk of postoperative bacterial endophthalmitis. In order to investigate whether the posterior capsule inhibited the spread of infection into the vitreous we performed extracapsular cataract extraction in both eyes of 10 primates. In one eye of each primate the posterior capsule was left intact and in the other eye a large posterior capsulectomy was performed. When the anterior chambers were challenged with equivalent inocula of Staphylococcus aureus, one of 10 eyes with an intact posterior capsule developed culture-positive .vitreous infection. In contrast, nine of 10 eyes with a large posterior capsulectomy developed culture-positive vitreous infection. In a second experiment we investigated the effect of an intraocular lens on the barrier effect. Ten primates received extracapsular cataract extraction in both eyes and pseudophakic implantation. In one eye of each primate the posterior capsule was left intact and a J-loop monoplanar lens was implanted in the ciliary sulcus. In the other eye of each primate a large posterior capsulectomy was followed by implantation of a monoplanar, non-vaulted pseudophakos into the anterior chamber. None of the 10 eyes with a posterior capsule intact and a posterior chamber lens in place developed positive vitreous cultures or histopathological evidence of vitreous infection. Thus the presence of a posterior chamber lens did not appreciably compromise the barrier effect of the intact posterior capsule. $40 \%$ of eyes with a large posterior capsulectomy and a non-vaulted pseudophakos in the anterior chamber developed culture-positive vitreous infection, and $60 \%$ of the eyes showed histopathological evidence of vitreous infection.

Unfortunately, scant attention has been paid in recent years to the prevention of postoperative bacterial endophthalmitis. Retrospective clinical studies suggested that preoperative topical antibiotics reduce the incidence of postoperative bacterial endophthalmitis. ${ }^{2}$ There is no scientific proof, however, that modern topical antibiotic regimens actually reduce the incidence of postoperative bacterial endophthalmitis. Some surgeons, therefore, do not use preoperative antibiotics. ${ }^{3}$

Other than these retrospective, uncontrolled studies which seemed to show that certain preopera-

\footnotetext{
*Presented in part at the American Academy of Ophthalmology Meeting, Chicago, Illinois, November 1983.

Correspondence to Francis E O'Donnell Jr, Md, 3663 Wendell Blvd, St Louis, Missouri 631 08, USA.
}

tive topical antibiotics can be effective in reducing the incidence of postoperative bacterial endophthalmitis there have been no quantitative studies demonstrating the clinical efficacy of other preventive measures. In part this is because such clinical trials would require a vast number of cases.

There has been a legitimate concern that the growing popularity of extracapsular cataract surgery could result in a rise in the number of cases of postoperative bacterial endophthalmitis. Multiple entries into the eye and the infusate are added sources of intraoperative contamination. There are reasons to suspect that extracapsular surgery which preserves the posterior capsule intact may be safer than if the posterior capsule is violated. Maylath and Leopold previously showed that in the phakic eye 
inoculation of bacteria into the anterior chamber was much less effective in producing bacterial endophthalmitis than was vitreous inoculation. ${ }^{+}$During an era when intracapsular cataract extraction was used in the vast majority of cases, Forster ${ }^{5}$ and others reported cases of postoperative bacterial endophthalmitis in which the anterior chamber culture was negative but the vitreous culture was positive, suggesting that the anterior chamber is somehow better able to control infection than is the vitreous cavity. From these observations we reasoned that the posterior capsule might act as a physical barrier to the spread of organisms into the vitreous cavity, and that if the organisms could be confined to the anterior chamber they might be more effectively overcome by the body's defense mechanisms. If this were the case, we considered that the clinical implications would be far-reaching.

\section{Material and methods}

\section{EXPERIMENT I}

In the first experiment we performed extracapsular cataract extraction in both eyes of 10 adult primates (rhesus and cynomologus). The details of the surgical technique are published elsewhere. ${ }^{6}$ The anterior capsulotomy was large, extending to the edge of the fully dilated pupil. The cortical clean-up was meticulous. In one eye of each primate the posterior capsule was left intact. In the other eye a large posterior capsulectomy (estimated to be at least $9 \mathrm{~mm}^{2}$ ) with no or only minimal anterior vitrectomy was performed with a vitrector inserted through the limbus. Minimal vitrectomy was performed if the anterior hyaloid was disrupted. To exclude intraoperative contamination we waited two weeks postoperatively before challenging the anterior chamber of each eye with an equivalent inoculum of Staphylococcus aureus.

Two weeks after surgery all eyes were found to be quiet, with clear media. Conjunctival chemosis and/or hyperaemia was absent. No eye had any clinical sign of endophthalmitis. The technique for preparation of the inocula has been reported in detail elsewhere. ${ }^{6}$ Appropriate dilutions were made accordingly to obtain $10^{3}$ or $10^{4}$ organisms per $0.05 \mathrm{ml}$ of saline. Microbial concentrations were verified at the time of preparation by inoculating known volumes of suspension on blood agar plates and performing counts after 24 hours.

After intramuscular injection of ketamine HCL a 'bevel-up injection' (that is, with the port of the needle facing towards the cornea and not towards the vitreous cavity) was carefully made into the anterior chamber of each monkey eye through the surgical wound, and $0.05 \mathrm{ml}$ was injected. After inoculation

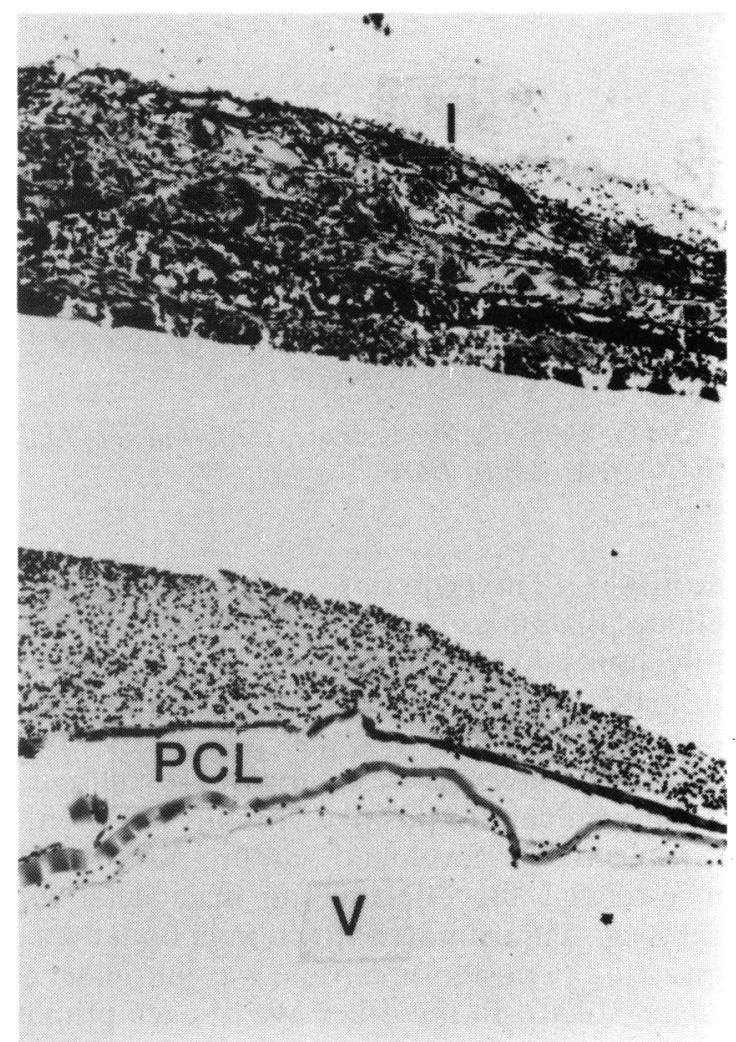

Fig. 1 Primate eye which had received extracapsular cataract extraction with intact posterior capsule and posterior chamber implant. The iris (I) is intensely infiltrated with inflammatory cells. The inflammatory exudate was layered on the anterior surface of the implant (PCL), which was dissolved in the paraffin embedding. The intact posterior capsule is present with a few inflammatory cells in the anterior vitreous $(V) . P C L=$ posterior chamber implant cavity, $I=$ iris, $V=$ vitreous. (Haematoxylin and eosin).

each animal was examined by handlight by two observers who had no previous knowledge of which eye had undergone capsulectomy. Their clinical examinations were recorded every 24 hours for 72 hours. At 72 hours after inoculation all animals were killed and the anterior chamber and central vitreous cavities were cultured separately by aseptic techniques. The anterior chamber was cultured by using a translimbal approach to aspirate $0.1 \mathrm{ml}$, and the central vitreous cavity was cultured by a pars plana approach to aspirate $0.5 \mathrm{ml}$. The specimens were immediately inoculated on to blood agar plates. Cultures were judged to be positive if even a single colony of phage-typed Straphylococcus aureus was isolated on the streak. The globes were then hemisected and the vitreous cavities were examined with the dissecting biomicroscope (Fig. 1). The 
primate organs were then used by other investigators at the St Louis University School of Medicine.

\section{EXPERIMENT 2}

In the second experiment we wanted to determine if the presence of a posterior chamber lens in any way affected the protective barrier effect of the intact posterior capsule. Moreover we investigated whether or not a non-vaulted monoplanar lens would províde some protective barrier effect to the spread of infection from the anterior chamber into the vitreous cavity. Extracapsular cataract extraction was performed in both eyes of 10 primates. In one eye of each of 10 primates the posterior capsule was left intact, and a J-loop monoplanar lens was inserted into the posterior chamber. It was our clinical impression that the lens was inserted into the ciliary sulcus and not into the capsular bag. In the other eye extracapsular cataract extraction was then followed by posterior capsulectomy (estimated to be at least $9 \mathrm{~mm}^{2}$ ) with minimal anterior vitrectomy by means of a vitrector through the limbus, and then a monoplanar lens (non-angulated J-loop lens) was inserted into the anterior chamber. No peripheral iridectomy was performed. Two weeks after the operation the anterior chambers were challenged with equivalent inocula of Staphylococcus aureus organisms, as in study $1.10^{4}$ organisms in $0.05 \mathrm{ml}$, as in study 1 , were injected into the anterior chamber of each eye. Clinical observations by handlight were made at 24hour intervals for 72 hours. At 72 hours the animals were killed, the anterior chambers were cultured with a translimbal aspiration of aqueous and with a pars plana aspiration of the central vitreous cavity, as in study 1 . The globes were then submitted for gross pathological and histopathological study.

\section{Results}

EXPERIMENT I (Table 1)

Monkey 1 had an inoculation of each eye with $10^{3}$ Staphylococcus aureus organisms and failed to develop infection in either eye. Therefore in the remaining primates we chose to use a dose of $10^{4}$ Staph. aureus organisms. None of the nine eyes which had an intact posterior capsule developed biomicroscopic evidence of vitreous inflammation. Nevertheless, one eye with an intact capsule had a positive vitreous culture for Staph. aureus (a single colony on the blood agar plate). All nine eyes with extensive posterior capsulectomy developed biomicroscopic evidence of vitreous inflammation, and all nine eyes had positive vitreous cultures for Staph. aureus. By Fisher's exact test the disparity between the two groups could have occurred by chance with a probability of less than $0 \cdot 00021$.
Table 1 Results of experiment 1

\begin{tabular}{lll}
\hline & Capsule intact & Capsulectomy \\
\hline No. of eyes & 10 cyes & 10 cyes \\
Anterior chamber culture positive & 5 & 5 \\
Vitreous culture positive* & 1 & 9 \\
Vitreous inflammation & 0 & 9 \\
Anterior chamber culture positive & 4 & 0 \\
and vitreous culture negative & & \\
\hline
\end{tabular}

${ }^{*} \mathrm{p}<0 \cdot 00021$

EXPERIMENT 2 (Table 2)

None of the 10 eyes with the posterior capsule intact and with the posterior chamber lens in place developed positive vitreous cultures. Moreover gross pathological and histopathological study of these specimens did not show evidence of vitreous infection. There was no significant spread of lens epithelium on to the posterior capsule. By contrast, in four of the 10 eyes with capsulectomy and pseudophakos in the anterior chamber there were positive vitreous cultures. Moreover in six of the eyes there was biomicroscopic and histopathological evidence of inflammation within the vitreous cavity (Fig. 2). Analysis of culture results by Fisher's exact test showed that the difference between the two groups was statistically significant at $p<0 \cdot 043$.

\section{Discussion}

We chose to challenge the experimental eyes with Staphylococcus aureus for two reasons. Firstly, Staph. aureus is an important cause of virulent postoperative bacterial endophthalmitis. ${ }^{7}$ Secondly, it is more virulent than some other bacteria responsible for postoperative bacterial endophthalmitis, such as Staph. epidermidis. We reasoned that if a protective barrier effect could be demonstrated for Staph. aureus, it was likely that this same barrier effect would exist for less virulent organisms. Certainly the visual prognosis for eyes with postoperative bacterial endophthalmitis due to Staph. aureus is much worse than for Staph. epidermiditis. ${ }^{\text {? }}$

Table 2 Results of experiment 2

\begin{tabular}{lll}
\hline & $\begin{array}{l}\text { Capsule intact } \\
\text { and posterior } \\
\text { chamberlens }\end{array}$ & $\begin{array}{l}\text { Capsulectomy } \\
\text { and anterior } \\
\text { chamberlens }\end{array}$ \\
\hline No. of cyes & 10 eyes & 10 eyes \\
Anterior chamber culture positive & 4 eyes & 7 eyes \\
Vitreous culture positive* & 0 & 4 \\
Vitreous inflammation & 0 & 6 \\
Anterior chamber culture positive & 4 & 3 \\
and vitrcous culture negative & & \\
\hline
\end{tabular}

${ }^{*} \mathrm{p}<0 \cdot \mathbf{0 4 3}$ 


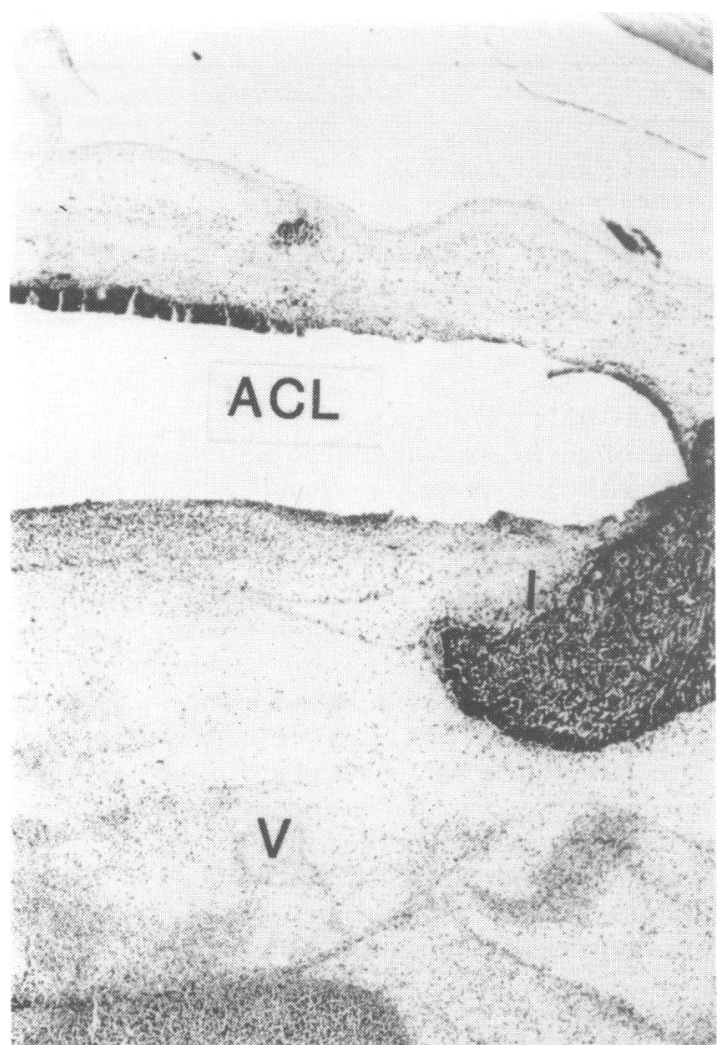

Fig. 2 Primate eye which had received extracapsular cataract extraction with posterior capsulectomy and anterior chamber implant (ACL). A massive inflammatory exudate surrounds the implant cavity with intense vitritis (V).

$A C L=$ anterior chamber implant cavity, $I=$ iris, $V=$ vitreous. (Haematoxylin and eosin).

The first primate experiment showed that the intact posterior capsule does have a protective barrier effect. It inhibits the spread of bacterial infection into the vitreous cavity. By helping to confine the infective organisms in the anterior chamber it allows the body's natural defence mechanisms to control the infection more adequately. Certainly the posterior capsule is a relative and not an absolute barrier to the spread of infection from the anterior chamber to the vitreous cavity. In the second animal experiment we showed that this protective barrier effect of the intact posterior capsule was not appreciably compromised by the presence of a posterior chamber lens which had been implanted into the ciliary sulcus. The clinical implications of these experimental primate studies are numerous.

Firstly, primary or secondary surgical posterior capsulotomies should be avoided if at all possible. In Hurite's series, for example, several patients developed bacterial endophthalmitis following secondary surgical posterior capsulotomy. ${ }^{\text {X }}$ Delayed non-invasive opening of the posterior capsule with a YAG laser is preferred, because it disrupts the barrier without a concomitant inoculation of bacteria. Other than superior ease of performance, this is at present the most compelling reason for using the YAG laser for posterior capsulectomy rather than surgical posterior capsulotomy, since the rates of other complications may not differ significantly, though Keates $e t$ al. have recently reported that the incidence of cystoid macular oedema may be less with YAG posterior capsulectomy. ${ }^{9}$ Since suture removal for irritation or to control astigmatism may be associated with bacterial endophthalmitis,' YAG posterior capsulectomy should be delayed until further suture removal is unlikely.

Secondly, cataract cases at particular risk for endophthalmitis, such as cases of penetrating corneal laceration and traumatic cataract, should have the posterior capsule preserved if at all possible. Translimbal vitrectors are often used to treat such cases, but unless the surgeon encounters formed vitreous he should studiously avoid indiscriminate removal of the posterior capsule.

Thirdly, in cases with filtering blebs, the risk for delayed postoperative endophthalmitis is significant (albeit related to the type of filtration bleb"' "), and the posterior capsule should be left intact if at all possible. Therefore in patients with filtration blebs who have developed significant cataracts we strongly recommend the use of extracapsular surgery, especially if the bleb is cystic and thin-walled. ${ }^{3}$ For these same reasons in patients undergoing combined cataract and filtration surgery we also recommend extracapsular surgery.

Fourthly, what if the posterior capsule eventually opacifies? Although this study did not investigate whether the size of the capsulotomy would have an influence on the protective barrier effect of the posterior capsule, it seems likely to us that small, centrally placed YAG capsulectomies are more desirable than large capsulectomies. If a posterior chamber lens is present, this may act to 'seal' the small, centrally placed posterior capsulectomy, as suggested by Simcoe (personal communication) and by our own studies. ${ }^{12}$ In a previous study we found that only one of five primate eys which had received extracapsular cataract extraction, posterior chamber implant, and a posterior capsulotomy developed endophthalmitis after the injection of $10^{4}$ Staph. aureus into the anterior chamber. In this eye the posterior capsulotomy extended inadvertently beyond the edge of the optic, whereas in the other four eyes the posterior capsulotomy had been smaller and did not extend beyond the edges of the optic. 
Therefore, if a secondary capsulotomy is necessary, we recommend the use of a small, centrally placed capsulectomy with the YAG laser. If a posterior chamber lens is present, the capsulectomy should not extend beyond the edges of the optic. In glaucomatous eyes with active filtration this also reduces the risk of vitreous prolapse into the filtration site.

Fifthly, the barrier effect of the intact posterior capsule means that we must begin to modify our approach to the postoperative patient who develops intraocular inflammation. It is important to note (see Tables 1 and 2) that some anterior chamber cultures were positive ( 7 out of 20 eyes) in the primate eyes with intact posterior capsule although the vitreous was culture negative. Once again this underscores the barrier effect of the intact capsule. It also suggests that we may begin to appreciate clinically a type of postoperative endophthalmitis which is confined to the anterior segment ('anterior endophthalmitis') and which does not require direct vitreous therapy. ${ }^{3}$ The combination of frequent topical antibiotics and intravenous antibiotics may suffice, especially when the organism is Staphylococcus epidermidis. ${ }^{13}$ In clinical practice we now examine such patients at the slit-lamp in order to study their anterior vitreous. In a series of over 500 extracapsular cataract extraction with posterior chamber implant and intact posterior capsule cases we have not personally seen a cellular response in the anterior vitreous prior to three weeks postoperatively, when some sterile anterior vitritis is occasionally visible on biomicroscopy. Therefore a cellular reaction in the vitreous of eyes with an intact posterior capsule within the first week postoperatively would be interpreted as a grave sign of possible infection, with spread of infected material into the vitreous, and our management would be more aggressive, including vitreous tap or vitrectomy and intravitreal antibiotics. Although it has been suggested that in the aphakic patient the vitreous cavity can be cultured through a translimbal approach following aspiration of aqueous, our studies strongly suggest that it would be unwise to culture the vitreous cavity through the limbus by penetrating the posterior capsule with a needle and aspirating vitreous. Such a manoeuvre would probably enhance the spread of any infected material into the vitreous cavity. In eyes with extracapsular cataract extraction and intact posterior capsule the vitreous cavity should be cultured by a pars plana approach.

Sixthly, when the posterior capsule is inadvertently disrupted, the risk of infection is greater. Unfortunately, when an extracapsular cataract extraction is complicated by vitreous loss, this usually occurs prior to removal of all cortex. In some series 'unplanned extracapsular cataract extraction' (that is, cataract extraction that has vitreous admixed with retained cortex) has a threefold higher risk of bacterial endophthalmitis than does an uncomplicated intracapsular cataract extraction.' We recommend immediate pars plana vitrectomy for complicated extracapsular cases with retained cortical material admixed with formed vitreous, and with significant intraocular inflammation, for three reasons: (1) there is an increased risk of bacterial endophthalmitis in this situation; (2) it can be virtually impossible early on to determine clinically whether or not this is sterile inflammation or infection; (3) the inflammation induced by the retained cortex in the vitreous reduces the visual potential. In Jaffe and Clayman's series, ${ }^{14}$ for example, the chances of cystoid macular oedema are significantly greater with vitreous loss in extracapsular cataract extraction than with vitreous loss in intracapsular cataract extraction. Pars plana vitrectomy reduces the inflammatory load of retained cortex. Although a pars plana vitrectomy has usually been reserved for management of bacterial endophthalmitis which is unresponsive to intravenous and intracameral antibiotics for the reasons just cited, we personally feel that a pars plana vitrectomy should be used promptly in complicated extracapsular cases with suspected endophthalmitis.

Seventhly, any intraocular manipulation in the anterior segment after cataract surgery carries the risk of bacterial endophthalmitis, so an intact posterior capsule is desirable. For example, in patients who elect not to have intraocular lens implantation at the time of cataract surgery, but who may later decide to have intraocular lens implantation because of difficulties with contact lenses or aphakic spectacles, extracapsular surgery with an intact posterior capsule would seem to reduce the chances of bacterial endophthalmitis during secondary implantation. This would be true regardless of whether the implant chosen was angle-supported or in the posterior chamber. A YAG posterior capsulectomy should be deferred if possible, until after it is clear that the patient can tolerate contact lenses or aphakic spectacles.

In conclusion, these experimental studies demonstrate yet another important effect of the intact posterior capsule. In addition to lowering the incidence of cystoid macular oedema ${ }^{14}$ and reducing the chances of rhegmatogenous retinal detachment, at least in axial myopes, ${ }^{\text {is }}$ the intact posterior capsule provides some protection against bacterial endophthalmitis after extracapsular surgery.

Supported in part by an unrestricted grant from Research to Prevent Blindness Inc. and by the Golden Fund.

\section{References}

1 Allen HF, Mangiaracine AB. Bacterial endophthalmitis after cataract extraction. Ophthalmology (Rochester) 1973; 77: 581-8. 
2 Christy NE. Lall P. Post-operative endophthalmitis following cataract surgery. Arch Ophthalmol 1973; 90: 361-6.

3 Spaeth GL, Weinstein GW, Kramer SG, Gutman FA, O'Day D. Prevention and treatment of ophthalmic infections. Ophthalmic Surg 1984; 15 (suppl): 9-19.

4 Maylath FR, Leopold IH. Study of experimental intraocular infection. Am J Ophthalmol 1955; 40: 86-101.

5 Forster RK. Endophthalmitis: diagnostic cultures and visual results. Arch Ophthalmol 1974; 92: 387-92.

6 Beyer TL, Vogler G, Sharma D, O'Donnell FE. Protective barrier effect of the posterior lens capsule in exogenous bacterial endophthalmitis. Invest Ophthalmol Vis Sci 1984; 25: 108-22.

7 Olson JC, Flynn HW, Forester RK, Culberson WW. Results in the treatment of post-operative endophthalmitis. Ophthalmology (Rochester) 1983; 90: 692-7.

8 Hurite FG. Intracapsular cataract extraction versus extracapsular cataract extraction. American Academy of Ophthalmology Regional Update Mecting, March 1982, Philadelphia, PA.

9 Keates RK, Steinert RF, Puliafito CA, Maxwell SK. Long-term follow-up of Nd:YAG laser posterior capsulotomy. Am Intraocular Implant Soc J 1984; 10: 164-8.

10 Pillai S, Limaye SR. Endophthalmitis following Schcic procedure. Glaucoma 1984; 6: 96-8.

11 Bellows AR, McCulley JP. Endophthalmitis in aphakic patients with unplanned filtering blebs wearing cataract lenses. Ophthalmology (Rochester) 1981; 88: 839-43.

12 Beyer TL, Vogler G, Sharma D, O’Donnell FE. Protective barrier effect of the posterior lens capsule in exogenous bacterial endophthalmitis: an experimental pseudophakic primate study. Am Intraocular Implant Soc J 1983; 9: 293-6.

13 O'Day DM, Jones DB, Patrinely J, Elliott JH. Staphylococcus cpidermidis endophthalmitis: visual outcome following noninvasive therapy. Ophthalmology (Rochester) 1982; 89: 354-60.

14 Jaffe NS, Clayman HM. Cystoid macular edema after intracapsular and extracapsular cataract extraction with or without an extraocular lens. Ophthalmology (Rochester) 1982; 89: 25-9.

15 Clayman HM, Jaffe WS, Light DS, Jaffe MS, Cassady JC. Intraocular lenses, axial length, and retinal detachment. $A m \mathrm{~J}$ Ophthalmol 1981; 92: 778-80. 